\title{
Marshfield Linkage Map
}

National Cancer Institute

\section{Source}

National Cancer Institute. Marshfield Linkage Map. NCI Thesaurus. Code C70943.

A microsatellite-based human genetic map based on 7740 microsatellite markers

originally identified at the Marshfield Clinic. 